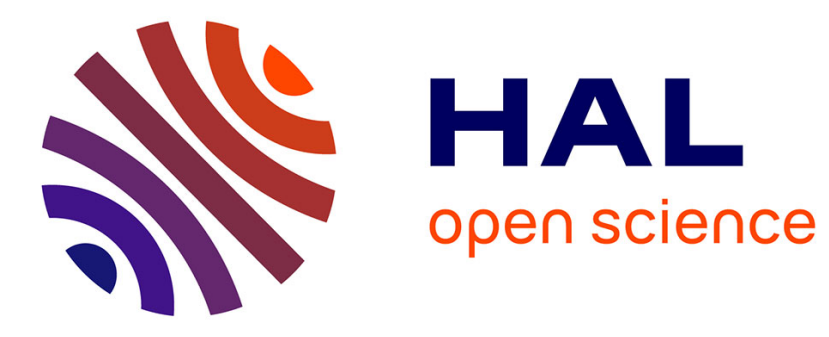

\title{
Insular lateralization in tinnitus distress
}

E van Der Loo, Marco Congedo, S Vanneste, P van de Heyning, D de Ridder

\section{To cite this version:}

E van Der Loo, Marco Congedo, S Vanneste, $\mathrm{P}$ van de Heyning, D de Ridder. Insular lateralization in tinnitus distress. Autonomic Neuroscience: Basic and Clinical, 2011, 165 (2), pp.191-4. 10.1016/j.autneu.2011.06.007 . hal-00643188

\section{HAL Id: hal-00643188 \\ https://hal.science/hal-00643188}

Submitted on 21 Nov 2011

HAL is a multi-disciplinary open access archive for the deposit and dissemination of scientific research documents, whether they are published or not. The documents may come from teaching and research institutions in France or abroad, or from public or private research centers.
L'archive ouverte pluridisciplinaire $\mathbf{H A L}$, est destinée au dépôt et à la diffusion de documents scientifiques de niveau recherche, publiés ou non, émanant des établissements d'enseignement et de recherche français ou étrangers, des laboratoires publics ou privés. 
1 Insular Lateralization in Tinnitus Distress.

2 van der Loo, E. ${ }^{1}$, Congedo, M. ${ }^{2}$, Vanneste, S. ${ }^{1}$, Van De Heyning, P. ${ }^{3}$, De Ridder, D. ${ }^{1}$

3

4 1. BRAI'N \& Department of Neurosurgery, University Hospital Antwerp, Belgium,

5 2. National Center for Scientific Research (CNRS), GIPSA-lab, Grenoble, France,

6 3. BRAI'N \& Department of ENT, University Hospital Antwerp, Belgium

7 
9 Tinnitus affects $15 \%$ of the population. Of these $1-2 \%$ are severely disabled by it. The role of 10 the autonomic system in tinnitus is hardly being investigated. The aim of this study is to 11 investigate the relationship between tinnitus distress and lateralization of the anterior insula, 12 known to be involved in interoceptive awareness and (para)sympathetic changes. For this, 13 Tinnitus Questionnaire scores are correlated to Heart Rate Variability markers, and related to 14 neural activity in left and right anterior insula. Our results show that tinnitus distress 15 is related to sympathetic activation, in part mediated via the right anterior insula. 


\section{Introduction}

Tinnitus is a symptom that affects $15 \%$ of the population (Axelsson and Ringdahl, 1989). Most people who have tinnitus can effectively cope with it, however a small percentage of tinnitus sufferers demonstrate maladaptive coping (Budd and Pugh, 1996; Scott et al., 1990; Tyler et al., 2006): $1-2 \%$ of tinnitus sufferers are severely disabled by their tinnitus (Axelsson and Ringdahl, 1989). This maladaptive coping group suffers significantly more from associated somatic complaints such as headaches, neck and shoulder pain, low back pain, muscle tension, sleep and concentration problems (Hiller et al., 1997; Scott and Lindberg, 2000) and demonstrates cognitive inefficiency (Hallam et al. 2004), poor stress coping (Scott and Lindberg 2000) and depression (Dobie, 2003; Folmer and Shi, 2004; Harrop-Griffiths et al., 1987; Scott and Lindberg, 2000; Sullivan et al., 1988).

The amount of distress people experience related to tinnitus can be evaluated by the use of validated tinnitus questionnaires. Tinnitus distress is associated to a higher orthosympathetic (OS) tone (Datzov et al., 1999) and tinnitus suppression induces an increased parasympathetic (PS) tone (Matsushima et al., 1996). Previous functional imaging studies show that specific frontal cortical areas closely relate to emotion perception and interoception. The right anterior insula seems to be specifically involved in the representation of subjective feelings (Craig, 2003; Critchley et al., 2004). Based on human lesion and electrical stimulation studies it has also been suggested that the right insula controls cardiac OS activity whereas the left insula is predominantly associated to PS activity (Oppenheimer, 1993, 2006; Oppenheimer et al., 1992; Oppenheimer et al., 1996). Functional Magnetic Resonance Imaging (fMRI) studies of sympathetic skin conductance response seem to confirm this lateralization by revealing right insula activation (Critchley et al., 2000). Furthermore, when correlating dichotic visual stimuli with Heart Rate Variability (HRV) the same lateralization effect is found (Wittling et al., 1998a; Wittling et al., 1998b).

Heart Rate Variability (HRV) is a simple and non-invasive quantitative marker of autonomic function. As a result of continuous variations of the balance between OS and PS neural activity influencing heart rate, intervals between consecutive heartbeats (RR intervals) show spontaneously occurring oscillations. For HRV spectral analysis three main underlying frequencies have been used in literature: the very-low-frequency range (VLF $\leq 0.04 \mathrm{~Hz}$ ), the low-frequency range (LF: $0.04-0.15 \mathrm{~Hz}$ ) and the high frequency range (HF: $0.15-0.4 \mathrm{~Hz}$ ). The high frequency component of HRV is believed to be influenced by vagal activity and is also related to the frequency of respiration (Yasuma and Hayano, 2004). Low-frequency (LF) power is modulated by baroreceptor activities and fluctuations in heart rate in the LF range reflect OS as well as PS influences. Low-frequency power, therefore, cannot be considered to reflect pure OS activity. However if normalized units of LF and HF are considered, the OS and PS influences respectively are emphasized (Electrophysiology, 1996). In HRV frequency domain, normalized units (n.u.) of LF and HF components therefore reflect OS and PS influences respectively. 
The aim of this study is to investigate the relation between tinnitus distress and lateralisation of the anterior insula, known to be involved in interoceptive awareness and OS as well as PS changes. For this, tinnitus questionnaire (TQ) scores (Goebel and Hiller, 1994) are correlated to HRV markers, and related to neural activity in left and right anterior insula.

\section{Methods}

Ten patients with strictly right-sided unilateral tinnitus are analyzed. EEG and ECG signals are recorded simultaneously over $5 \mathrm{~min}$ in supine position using a 32 channel digital EEG (Neuroscan, Compumedics, Houston, TX) in a dimly illuminated and soundproof room (sampling rate $=500 \mathrm{~Hz}$, band passed $0.15-100 \mathrm{~Hz}$ ). Electrodes are referenced near the vertex and impedances checked to remain below $5 \mathrm{k} \Omega$. To minimize respiratory influences on HRV, respiration is controlled at 12 beats per minute using auditory cues. All patients complete a validated Dutch version of the TQ (Meeus et al., 2007), which reflects the amount of tinnitus related distress perceived by the patient (Goebel and Hiller, 1994).

\section{ECG analyses}

ECG signals are processed by time and frequency domain methods as recommended by the Task force (Electrophysiology, 1996): QRS complexes are recognized from the short-term artifact-free ECG recordings from which peaks (R-waves) are detected and from which intervals between two consecutive peaks (RR intervals) are calculated. Once HRV time series are extracted they are analyzed in the time and frequency domain using HRV Analysis Software 1.1 for windows developed by The Biomedical Signal Analysis Group, Department of Applied Physics, University of Kuopio, Finland. Pearson correlations between OS (LF n.u.) and PS (HF n.u.) markers of HRV and TQ-scores are performed.

\section{EEG analyses}

EEG segments contaminated by artifacts are rejected offline by visual inspection. The remaining data are analyzed in the frequency domain by means of Fast Fourier Transform (FFT) analysis. Two regions of interest (ROI) corresponding to the right and left anterior insula are selected in the $\mathrm{MNI}$ atlas (Fig. 1). A spatial filter approach known as beamforming (Congedo, 2006) targeting these two ROIs is applied in order to obtain current density estimations within these ROls by the eLORETA method (Pascual-Marqui 2007). The logcurrent density is correlated with the TQ-scores, in all $1 \mathrm{~Hz}$ spaced discrete Fourier frequencies in the range $1 \mathrm{~Hz}-60 \mathrm{~Hz}$. Significant trends are formulated with a $p<0.05$.

Fig. 1: about here.

\section{Results}

TQ-scores $(M=40.2 ; S D=13.7)$ correlate positively with the OS marker, the Low Frequency normalized units $(r=0.58)$, and negatively with the PS marker, the High Frequency normalized units $(r=-0.58)$. 
In addition, current density analyses show that increased cortical activity in the left anterior insula at $11 \mathrm{~Hz}(r=0.56$; alpha band $)$ and decreased activity at $4 \mathrm{~Hz}(r=-0.63$; theta band $)$ and in the high gamma band frequencies $(54 \mathrm{~Hz}, r=-0.58 ; 59 \mathrm{~Hz}, r=-0.74)$ relates to increased TQ-scores. In the right anterior insula increasing TQ-scores were found with increased activity in delta band frequencies $(2 \mathrm{~Hz}, r=0.67)$ and gamma band frequencies $(32 \mathrm{~Hz}, r=0.74 ; 39 \mathrm{~Hz}$, $r=0.56)$ no significant decreases are noted in this area.

\section{Discussion}

Our results show a positive relation between OS load and tinnitus distress as measured by the TQ (Goebel and Hiller, 1994). In addition the right anterior insula, an area related to OS influence, shows increased delta and gamma activity related to increased tinnitus distress. On the other hand decreased theta and gamma activity are found in the left anterior insula, an area related to PS influence.

At a resting state the sensory cortices are characterized by alpha activity, which has been proposed to be an idling rhythm or a rhythm reflecting active inhibitory mechanisms (Klimesh et al., 2007). Gamma band activity is noted focally and waxes and wanes as it arises as a response to external stimuli, both in the visual (Crick \& Koch, 1990), auditory (Joliot et al., 1994) and somatosensory (Gross et al., 2007) system and thus reflects the activation of a cortical area. We suggest this mechanism can be extended to the autonomic nervous system. Gamma frequencies in this study increase or decrease together with low frequencies in the right or left anterior insula respectively, suggesting some type of nesting or coupling of high frequencies on low frequencies. Low frequencies (delta and theta) are widely distributed and activate larger networks (Gollo et al., 2010) and the nesting of gamma on theta or delta allows synchronization of widely distributed focal gamma activations, providing a mechanism for effective communication between these distributed areas (Canolty et al., 2006). Increasing distress, as measured by the $T Q$, is associated with an increase of alpha in the left insula and a decrease in theta and gamma, suggesting the left insula is actively inhibited by increasing distress, by the same alpha oscillation based mechanism encountered in other (sensory) cortices (Weisz et al., 2011). The delta and gamma activity in the right insula suggests this area is activated and associated with increasing distress.

The right insula has been related to interoception (Craig, 2003; Critchley et al., 2004; Taylor et al., 2008) and OS control (Oppenheimer, 1993, 2006; Oppenheimer et al., 1992). Damage to the left insula in humans can shift cardiovascular balance towards increased basal OS tone (Oppenheimer et al., 1996) and stimulation of the human right insula increases OS cardiovascular tone, whereas left insula stimulation increases parasympathetic (PS) tone (Oppenheimer, 1993). The right insula could therefore very well generate the subjective feelings of distress, i.e. the anxiety, associated with autonomic activity.

Many patients mention that tinnitus has developed in a stressful life episode and that it is worsened by stressful situations (Budd and Pugh, 1996; Hebert and Lupien, 2007). Tinnitus shares common pathophysiological, clinical and treatment characteristics with pain (De 
Ridder et al., 2007; Moller, 2000; Tonndorf, 1987) and the same observation is made in patients suffering from pain (Price, 2000).

In patients suffering posttraumatic stress disorder (PTSD) the prevalence of tinnitus is $50 \%$ (Hinton et al., 2006) and in soldiers presenting tinnitus 34\% also suffer from PTSD (Fagelson, 2007). This prevalence is much higher than in the normal population, where it is $10-15 \%$ (Axelsson and Ringdahl, 1989) suggesting a relation between tinnitus and some types of stress related disorders.

Former studies have also shown that stellate ganglion blocks can sometimes improve tinnitus transiently (Adlington and Warrick, 1971; Matoba et al., 1984; Warrick, 1969). The stellate ganglion is a sympathetic ganglion, thus suggesting the OS system could be a possible target for tinnitus treatment. Exploring potential central mechanisms of sympathetically mediated modulation of tinnitus therefore seems mandated.

At a cortical level, a Magnetoencephalographic (MEG) study demonstrated that tinnitus related distress is associated with a right sided connectivity increase between the anterior cingulate and the frontal cortex and parietal cortex (Schlee et al., 2008). However due to the technique used in this study it cannot be discerned which area of the frontal cortex is involved. On the other hand, a recent EEG study also showed that tinnitus distress involves a network which encompasses the amygdala, anterior cingulate, insula and parahippocampal area (Vanneste et al., 2010) although no lateralization effect was investigated.

In conclusion, this study suggests that tinnitus distress is related to OS activation, in part mediated via the right anterior insula, via spontaneous gamma and delta band activity as well as PS influence. Left insular alpha activity, suggesting PS inactivation, is correlated with with associated decreased theta and gamma activity. These data extend the concept that tinnitus distress is related to autonomic changes in the sympathetico-vagal balance, mediated at least in part by right sided anterior insular activity. The coupled low-high frequency changes suggest that the left insular gamma decrease and right insular gamma increase might be part of a larger theta based central autonomic nervous system network. This is also consistent with previous MEG and EEG studies investigating the neural correlates of tinnitus distress.

\section{Acknowledgments}

This study is performed with a grant from the American Tinnitus Association. The Tinnitus Research Initiative and St. Jude Medical Neurodivision are acknowledged for their support. The funders had no role in study design, data collection and analysis, decision to publish, or preparation of the manuscript. 
Axelsson, A., and Ringdahl, A. (1989). Tinnitus--a study of its prevalence and characteristics. Br J Audiol 23, 53-62.

Adlington, P., Warrick, J. (1971) Stellate ganglion block in the management of tinnitus. J Laryngol Otol 85: 159-168.

Budd, R.J., and Pugh, R. (1996). Tinnitus coping style and its relationship to tinnitus severity and emotional distress. J Psychosom Res 41, 327-335.

Canolty, R. T., Edwards, E., et al. (2006). High gamma power is phase-locked to theta oscillations in human neocortex. Science 313(5793), 1626-8.

Congedo, M. (2006). Subspace Projection Filters for Real-Time Brain Electromagnetic Imaging. IEEE Transactions on Biomedical Engineering, 53(8), 1624-34. Craig, A.D. (2003). Interoception: the sense of the physiological condition of the body. Current opinion in neurobiology 13, 500-505.

Crick, F. and Koch, C. (2003). A framework for consciousness. Nat Neurosci. 6(2): p. 119-26. Critchley, H.D., Elliott, R., Mathias, C.J., Dolan, R.J. (2000). Neural activity relating to generation and representation of galvanic skin conductance responses: a functional magnetic resonance imaging study. J Neurosci 20: 3033-3040.

Critchley, H.D., Wiens, S., Rotshtein, P., Ohman, A., and Dolan, R.J. (2004). Neural systems supporting interoceptive awareness. Nature neuroscience 7, 189-195.

Datzov, E., Danev, S., Haralanov, H., Naidenova, V., Sachanska, T., and Savov, A. (1999). Tinnitus, heart rate variability, and some biochemical indicators. Int Tinnitus J 5, 20-23.

De Ridder D, De Mulder G, Menovsky T, Sunaert S, Kovacs S (2007). Electrical stimulation of auditory and somatosensory cortices for treatment of tinnitus and pain. Prog Brain Res 166: 377-388.

Dobie, R.A. (2003). Depression and tinnitus. Otolaryngol Clin North Am 36, 383-388.

Electrophysiology, Task Force of the European Society of Cardiology the North American Society of Pacing Electrophysiology (1996). Heart rate variability: standards of measurement, physiological interpretation and clinical use. (Circulation), pp. 1043-1065. Fagelson, M.A. (2007). The association between tinnitus and posttraumatic stress disorder. Am J Audiol 16, 107-117. Folmer, R.L., and Shi, Y.B. (2004). SSRI use by tinnitus patients: interactions between depression and tinnitus severity. Ear Nose Throat J 83, 107-108, 110, 112 passim. Goebel, G., and Hiller, W. (1994). The tinnitus questionnaire. A standard instrument for grading the degree of tinnitus. Results of a multicenter study with the tinnitus questionnaire. Hno 42, 166-172. Gollo, L.L., Mirasso, C., Villa, A.E. (2010). Dynamic control for synchronization of separated cortical areas through thalamic relay. Neuroimage 52(3):947-55.

204 Gross, J., Schnitzler, A., Timmermann, L., Ploner, M. (2007). Gamma oscillations in human primary somatosensory cortex reflect pain perception. PLoS Biol 5: e133. 
Audiol 43, 218-226.

Hasson, D., Theorell, T., Wallén, M., Leineweber, C., Canlon, B. (2011). Stress and prevalence of hearing problems in the Swedish working population. BMC Public Health.

210 Harrop-Griffiths, J., Katon, W., Dobie, R., Sakai, C., and Russo, J. (1987). Chronic tinnitus:

211 association with psychiatric diagnoses. J Psychosom Res 31, 613-621.

212 Hebert, S., and Lupien, S.J. (2007). The sound of stress: Blunted cortisol reactivity to 213 psychosocial stress in tinnitus sufferers. Neurosci Lett 411: 138-142.

214 Hiller, W., Janca, A., and Burke, K.C. (1997). Association between tinnitus and somatoform 215 disorders. J Psychosom Res 43, 613-624.

216 Hinton, D.E., Chhean, D., Pich, V., Hofmann, S.G., Barlow, D.H. (2006). Tinnitus among 217 Cambodian refugees: relationship to PTSD severity. J Trauma Stress 19: 541-546.

218 Joliot, M., Ribary, U., and Llinas, R. (1994) Human oscillatory brain activity near $40 \mathrm{~Hz}$ 219 coexists with cognitive temporal binding. Proc Natl Acad Sci U S A. 91(24): p. 11748-51.

220 Klimesch, W, Sauseng, P., Hanslmayr, S. (2007). EEG alpha oscillations: the inhibition-

221 timing hypothesis, Brain Res. Rev. 53, 63-88.

222 Matoba, T., Noguchi, I., Noguchi, H., Sakurai, T. (1984). Stellate ganglion block for the relief 223 of tinnitus in vibration disease. Kurume Med J 31: 295-300.

224 Matsushima, J.I., Kamada, T., Sakai, N., Miyoshi, S., Uemi, N., and Ifukube, T. (1996). 225 Increased Parasympathetic Nerve Tone in Tinnitus Patients Following Electrical Promontory 226 Stimulation. Int Tinnitus J 2, 67-71.

227 Meeus, O., Blaivie, C., and Van de Heyning, P. (2007). Validation of the Dutch and the 228 French version of the Tinnitus Questionnaire. B-Ent 3 Suppl 7, 11-17.

229 Moller, A.R. (2000). Similarities between severe tinnitus and chronic pain. J Am Acad Audiol 230 11: 115-124.

231 Oppenheimer, S. (1993). The anatomy and physiology of cortical mechanisms of cardiac 232 control. Stroke 24, I3-5.

233 Oppenheimer, S. (2006). Cerebrogenic cardiac arrhythmias: cortical lateralization and clinical 234 significance. Clin Auton Res 16, 6-11.

235 Oppenheimer, S.M., Gelb, A., Girvin, J.P., and Hachinski, V.C. (1992). Cardiovascular effects 236 of human insular cortex stimulation. Neurology 42, 1727-1732.

237 Oppenheimer, S.M., Kedem, G., and Martin, W.M. (1996). Left-insular cortex lesions perturb 238 cardiac autonomic tone in humans. Clin Auton Res 6, 131-140.

239 Pascual-Marqui, R.D. (2007). Discrete, 3D distributed, linear imaging methods of electric 240 neuronal activity. Part 1: exact, zero error localization. http://arxiv.org/pdf/0710.3341

241 Price, D.D. (2000). Psychological and neural mechanisms of the affective dimension of pain.

242 Science 288: 1769-1772.

243 Schlee, W., Weisz, N., Bertrand, O., Hartmann, T., and Elbert, T. (2008). Using auditory 244 steady state responses to outline the functional connectivity in the tinnitus brain. PLoS ONE 3 , 245 e3720.

246 Scott, B., and Lindberg, P. (2000). Psychological profile and somatic complaints between 
247 help-seeking and non-help-seeking tinnitus subjects. Psychosomatics 41, 347-352.

248 Scott, B., Lindberg, P., Melin, L., and Lyttkens, L. (1990). Predictors of tinnitus discomfort, 249 adaptation and subjective loudness. $\mathrm{Br} \mathrm{J}$ Audiol 24, 51-62.

250 Sullivan, M.D., Katon, W., Dobie, R., Sakai, C., Russo, J., and Harrop-Griffiths, J. (1988).

251 Disabling tinnitus. Association with affective disorder. Gen Hosp Psychiatry 10, 285-291.

252 Taylor, K.S., Seminowicz, D.A., and Davis K.D. (2008). Two systems of resting state 253 connectivity between the insula and cingulate cortex. Hum Brain Mapp

254 Tonndorf, J. (1987). The analogy between tinnitus and pain: a suggestion for a physiological 255 basis of chronic tinnitus. Hear Res 28: 271-275.

256 Tyler, R.S., Coelho, C., and Noble, W. (2006). Tinnitus: standard of care, personality

257 differences, genetic factors. ORL J Otorhinolaryngol Relat Spec 68, 14-22.

258 Vanneste, S., Plazier, M., van der Loo, E., Van de Heyning, P., Congedo, M., and De Ridder, 259 D. (2010). The neural correlates of tinnitus-related distress. Neuroimage 52, 470-480.

260 Warrick, J.W. (1969). Stellate ganglion block in the treatment of Meniere's disease and in the 261 symptomatic relief of tinnitus. Br J Anaesth 41: 699-702.

262 Weisz, N., Hartmann, T., Müller, N., Lorenz, I., and Obleser, J. (2011). Alpha rhythms in 263 audition: cognitive and clinical perspectives. Front Psychol 2: 73

264 Wittling, W., Block, A., Genzel, S., and Schweiger, E. (1998a). Hemisphere asymmetry in 265 parasympathetic control of the heart. Neuropsychologia 36, 461-468.

266 Wittling, W., Block, A., Schweiger, E., and Genzel, S. (1998b). Hemisphere asymmetry in 267 sympathetic control of the human myocardium. Brain Cogn 38, 17-35.

268 Yasuma, F., and Hayano, J. (2004). Respiratory sinus arrhythmia: why does the heartbeat 269 synchronize with respiratory rhythm? Chest $125,683-690$ 
272 Weisz, N., T. Hartmann, et al. "Alpha rhythms in audition: cognitive and clinical perspectives."

273 Front Psychol 2: 73.

274

275

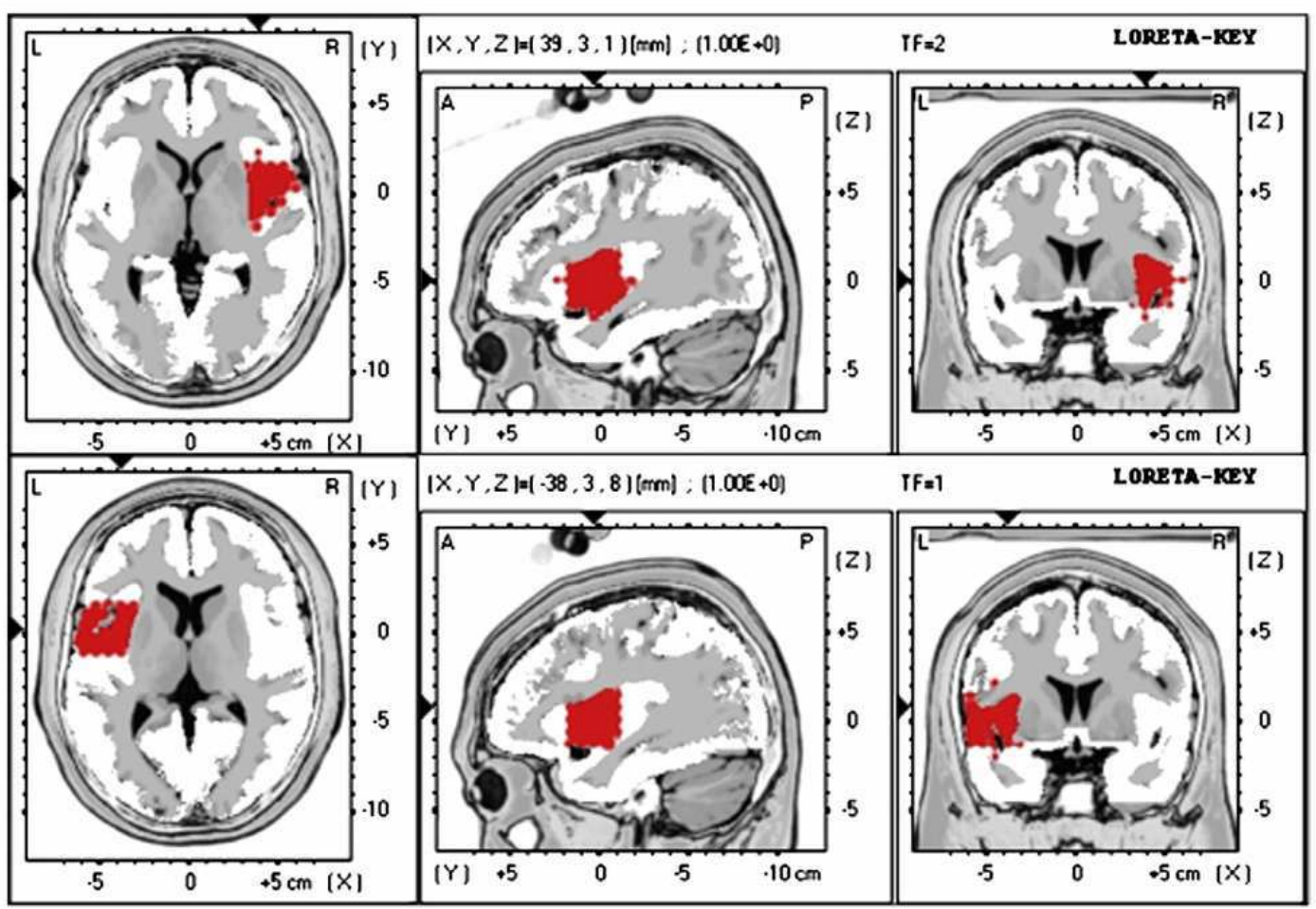

277 Fig. 1: Regions of interest: Right anterior insula (upper pannel) and left anterior insula 278 (lower pannel). Displayed sections are the axial (left), sagittal (middle), and coronal 279 (right) sections. 